\title{
Electrical Lead Locking Type
}

National Cancer Institute

\section{Source}

National Cancer Institute. Electrical Lead Locking Type. NCI Thesaurus. Code C111191.

The type of locking device used to securely fasten the electrical leads to the device. 